\title{
High-resolution X-ray spectroscopy of the Seyfert 1 Mrk 841: insights into the warm absorber and warm emitter
}

\author{
A. L. Longinotti ${ }^{1,2}$, E. Costantini ${ }^{3}$, P. O. Petrucci ${ }^{4}$, C. Boisson ${ }^{5}$, M. Mouchet ${ }^{5,6}$, M. Santos-Lleo ${ }^{2}$, G. Matt $^{7}$, \\ G. Ponti ${ }^{6}$, and A. C. Gonçalves ${ }^{5,8}$ \\ ${ }^{1}$ MIT Kavli Institute for Astrophysics and Space Research, 77 Massachusetts Avenue, NE80-6011, Cambridge, MA, 02139, USA \\ e-mail: annalia@space.mit.edu \\ 2 ESAC - European Space Astronomy Centre, PO Box 78, 28691 Villanueva de la Cañada, Madrid, Spain \\ SRON National Institute for Space Research, Sorbonnelaan 2, 3584 CA Utrecht, The Netherlands \\ ${ }^{4}$ Laboratoire d'Astrophysique, UMR 5571, Université J. Fourier/CNRS, Observatoire de Grenoble BP 53, 38041 Grenoble Cedex 9 , \\ France \\ 5 LUTH, UMR 8102, Observatoire de Paris, CNRS, Université Paris Diderot, 5 Place Jules Janssen, 92190 Meudon, France \\ 6 APC, UMR 7164, Université Paris 7 Denis Diderot, 75205 Paris Cedex 13, France \\ 7 Dipartimento di Fisica, Università degli Studi Roma Tre, Via della Vasca Navale 84, 00146 Roma, Italy \\ 8 CAAUL, Observatório Astronómico de Lisboa, Tapada da Ajuda, 1349-018 Lisboa, Portugal
}

Received 17 July 2009 / Accepted 12 November 2009

\section{ABSTRACT}

\begin{abstract}
Context. The Seyfert 1 galaxy Mrk 841 was observed five times between 2001 and 2005 by the XMM-Newton X-ray observatory. The source is well known for showing spectral complexity in the variable iron line and in the soft X-ray excess.

Aims. The availability of multiple exposures obtained by the reflection grating spectrometer (RGS) cameras allows thorough study of the complex absorption and emission spectral features in the soft X-ray band. This paper reports on the first study of Mrk 841 soft $\mathrm{X}$-ray spectrum at high spectral resolution.

Methods. The three combined exposures obtained in January 2001 and the two obtained in January and July 2005 were analysed with the SPEX software.

Results. We detect a two-phase warm absorber. A medium ionisation component $\left(\log \xi \sim 1.5-2.2 \mathrm{erg} \mathrm{s}^{-1}\right)$ is responsible for a deep absorption feature in the unresolved transition array of the Fe M-shell and for several absorption lines in the OVI-VIII band, and a higher ionisation phase with $\log \xi \sim 3 \mathrm{erg} \mathrm{s} \mathrm{cm}^{-1}$ is required to fit absorption in the NeIX-X band. The ionisation state and the column density of the gas present moderate variation from 2001 to 2005 for both phases. The high ionisation component of the warm absorber has no effect on the Fe $K$ band. No significant velocity shift of the absorption lines is measured in the RGS data. Remarkably, the 2005 spectra show emission features consistent with photoionisation in a high-density $\left(n_{\mathrm{e}} \geq 10^{11} \mathrm{~cm}^{-3}\right)$ gas. A prominent OVII line triplet was clearly observed in January 2005 and narrow radiative recombination continua (RRC) of OVII and CVI were observed in both 2005 data sets. A broad Gaussian line around $21.7 \AA$ was also required to fit all the data sets. The derived radial distance for the emission lines seems to suggest that the photoionisation takes place within the optical broad line region of the source.
\end{abstract}

Key words. galaxies: Seyfert - galaxies: individual: Mrk 841 - techniques: spectroscopic

\section{Introduction}

The model that explains active galactic nuclei (AGN) as being powered by accretion on a supermassive black hole surrounded by gas (Rees 1984) is nowadays widely accepted. In the past decade, the advent of high-resolution X-ray spectroscopy has allowed detailed study of ionised material flowing out along the line of sight within the central region (e.g. Kaspi et al. 2001; Kaastra et al. 2002). Multi-wavelength simultaneous observations have shown that X-ray outflows and UV winds are tightly connected and that the absorption must take place in a material with multiple ionisation layers (Gabel et al. 2005). More than $50 \%$ of the Seyfert 1 galaxies are characterised by this gas, which is mostly revealed in the X-ray band through detection of narrow absorption features from ionised elements, such as carbon, oxygen, neon, iron (Crenshaw et al. 2003; Blustin et al. 2005).

The structure of the outflowing wind and its relation to the central engine where the AGN continuum radiation originates, are not entirely clear. The absorbing gas seems to be distributed diversely in individual sources, its location spanning a scale of light days in, e.g., NGC 4051 (Krongold et al. 2007), to a few pc in, e.g., NGC 3783 (Behar et al. 2003).

Variability in the ionised absorbers can often offer a key to studying the change in the opacity of the gas in relation to the continuum emission. For example, long Chandra observational campaigns on bright Seyfert Galaxies have yielded a variety of results on the response of the warm absorber to the source luminosity fluctuations (Netzer et al. 2002; Krongold et al. 2005).

More recent studies have shown that the presence of emission lines in the soft X-ray band brings additional information on the circumnuclear ionised gas. In some Seyfert 1s, emission lines from highly ionised species can originate very close to or within the broad line region of the AGN (Costantini et al. 2007; Longinotti et al. 2008), but only in sources with high signal-tonoise has it been possible to relate the X-ray emitter with the X-ray absorber (NGC 3783, Behar et al. 2003).

Mrk 841 is a bright Seyfert 1 galaxy at $z=0.0365$ (Véron-Cetty \& Véron 2001) that has been observed by several $\mathrm{X}$-ray observatories in the past. Spectral variability was reported 
Table 1. XMM-Newton observation log for Mrk 841.

\begin{tabular}{ccccc}
\hline \hline $\begin{array}{c}\text { Date } \\
\text { (yyyy/mm/dd) }\end{array}$ & $\begin{array}{c}\text { OBSID } \\
-\end{array}$ & $\begin{array}{c}\text { Exp } \\
(\mathrm{ks})\end{array}$ & $\begin{array}{c}{ }^{1} \text { count rate } \\
\mathrm{Cts} / \mathrm{s}\end{array}$ & $\begin{array}{c}{ }^{2} F_{0.3-2 \mathrm{keV}} \\
\left(10^{-12} \mathrm{cgs}\right)\end{array}$ \\
\hline $2001 / 01 / 13$ & 0112910201 & 10 & $0.63 \pm 0.01$ & $26.6 \pm 1.0$ \\
$2001 / 01 / 13$ & 0070740101 & 12 & $0.72 \pm 0.01$ & $26.6 \pm 1.0$ \\
$2001 / 01 / 14$ & 0070740301 & 14 & $0.71 \pm 0.01$ & $26.6 \pm 1.0$ \\
$2005 / 01 / 16$ & 0205340201 & 45 & $0.19 \pm 0.01$ & $7.5 \pm 0.5$ \\
& & & & \\
$2005 / 07 / 17$ & 0205340401 & 30 & $0.23 \pm 0.01$ & $9.1 \pm 0.1$ \\
\hline
\end{tabular}

Notes. ${ }^{(1)}$ In RGS 2. ${ }^{(2)}$ Not corrected for absorption; the 2001 flux refers to the global fit of 3 data sets.

in the EXOSAT, Ginga and ROSAT data by George et al. (1993), and by Nandra et al. (1995). Evidence for a broad iron emission line was found in the ASCA spectra reported by Nandra et al. (1997). Weaver et al. (2001) have reported marginal evidence for line variability. The analysis of broad band BeppoSAX data revealed a soft X-ray excess and a Compton reflection component (Bianchi et al. 2001, 2004), although these authors found that warm absorption provided an equally good description of the soft X-ray data. Evidence for a moderately deep OVII absorption edge was also reported in ASCA data by Reynolds (1997). $X M M-N e w t o n$ data at CCD resolution highlighted the presence of a variable and complex narrow $\mathrm{Fe} \mathrm{K} \alpha$ line (Petrucci et al. 2002; Longinotti et al. 2004; Petrucci et al. 2007). Besides the study of Fe $K$ band, the later paper focused on the analysis of the soft excess in Mrk 841 by testing two competing models. On one hand, the soft excess could be modelled by reflection off a photoionised accretion disc whose spectrum is relativistically blurred and smeared (Crummy et al. 2006). A valid alternative model was proposed by Gierlinski \& Done (2004) who explained the soft excess as arising from ionised absorption in a relativistically smeared wind. According to Petrucci et al. (2007), both models were statistically indistinguishable in the EPIC data of Mrk 841.

This paper presents the very first analysis of the highresolution soft X-ray spectra of Mrk 841, and it makes use of all the available $X M M-N e w t o n$ data sets, i.e., the five observations previously studied by Petrucci et al. (2007).

\section{The XMM-Newton observations}

Mrk 841 was observed by XMM-Newton 3 times in 2001 and twice in 2005 (Table 1). In this paper, we present only the highresolution spectra gathered by the RGS instrument (den Herder et al. 2001). For more details on the EPIC data and on the broadband spectrum, the reader is referred to Petrucci et al. (2007). All the raw data were processed with SAS version 7.0.0. Spectral files for source and background were produced by running the standard procedure rgsproc. Response matrices were created by the task rgsrmfgen. The observation performed in January 2005 presents an anomaly in the illumination of CCD 2 (for both RGS1 and RGS2). The background subtraction was checked and confirmed as unaffected by this event.

The source varied significantly in flux and spectral shape over the time covered by XMM-Newton observations (see Table 1 and see Petrucci et al. 2007 for an extensive discussion on the variability properties of the source). The soft X-ray flux decreased by a factor of $\sim 3.5$ from 2001 to 2005, thus it is necessary to consider these two "epochs" separately.
The three short exposures of 2001 were checked carefully to decide whether the six RGS1 and RGS2 could be combined in two spectra (one for each instrument). We fitted the spectra with the same model to check the agreement in the spectral shape and in the flux level. No significant variation is found in the spectral shape in the RGS band, but since the count rates for the 2001 exposures differ by a few percent (see Table 1), instead of coadding the data, we decided to fit simultaneously the six spectra of 2001 assuming the same fitting model and normalising the data to the RGS1 in observation 0112910201.

This approach is maintained throughout the paper, and we refer to the 2001 simultaneously fitted spectra simply as "Jan. 2001". Since the exposures of 2005 are separated by 6 months, we checked whether they could be combined into a single one. After fitting the two 2005 spectra with a power law, the spectral slopes were found to be different by $\sim 20 \%$, therefore the 2005 spectra were not co-added in the analysis.

In summary, the three epoch spectra are kept disjointed during the entire spectral analysis and are referred to as Jan. 2001, Jan. 2005, and July 2005 throughout the paper.

\section{RGS spectral analysis}

The RGS spectral analysis was carried out by using the latest version of the fitting package SPEX (Kaastra et al. 1996) ${ }^{1}$. Galactic absorption of column density $N_{\mathrm{H}}=2.7 \times 10^{20} \mathrm{~cm}^{-2}$ was included in all the following spectral fits (Dickey \& Lockman 1990). The galactic absorption was modelled with the SPEX HOT component with a temperature fixed to $5 \times 10^{-4} \mathrm{keV}$. For all spectral models, we adopted Solar elemental abundances (Anders \& Grevesse 1989).

The first order spectra were rebinned of a factor of 3 over the range 7 to $35 \AA$ and the C-statistics was adopted (Cash 1979). Errors are quoted at the $90 \%$ confidence level for one interesting parameter. Throughout the text and tables, the quoted wavelengths have been corrected for the cosmological redshift.

\subsection{The warm absorber}

At first glance, visual inspection of the fluxed spectra (i.e. the spectrum plotted in flux units rather than counts/sec) reveals the typical signatures of absorption from the so-called unresolved transition array of the Fe M shell in the 15-16 $\AA$ band (e.g. Sako et al. 2001). The feature appears to be prominent in Jan. 2005, but it is also easily recognised in the other two data sets. We checked the residuals after fitting the spectra with a simple power law, confirming absorption in all of them, although with different characteristics. This may suggest variability in the ionised material along the three epochs and/or the presence of a multi-component warm absorber. In order to compare the three spectra and test this hypothesis, we took the approach of initially fitting them with the same model.

We constructed a warm absorber model with the SPEX builtin model XABS. The ionisation balance was calculated by assuming the spectral energy distribution (SED) of Mrk 841 as the ionising continuum spectrum. The SED was constructed with the simultaneous optical-UV-to-X-ray fluxes extracted from the optical monitor and the EPIC instruments onboard XMM-Newton. For the 2001 data set the photometric point in the U band (centred at $344 \mathrm{~nm}$ ) was used. In Jan. 2005 and July 2005, the source was observed with the optical grism, which produces a broader

\footnotetext{
1 See also http://www. sron.nl/spex
} 
Table 2. Best-fitting parameters for the two-phase warm absorber.

\begin{tabular}{ccccccccc}
\hline \hline $\begin{array}{c}\text { Obs } \\
-\end{array}$ & $\begin{array}{c}\Gamma_{\text {soft }} \\
-\end{array}$ & $\begin{array}{c}\log \xi \\
\left(\mathrm{erg} \mathrm{s} \mathrm{cm}^{-1}\right)\end{array}$ & $\begin{array}{c}N_{\mathrm{H}} \\
10^{21} \mathrm{~cm}^{-2}\end{array}$ & $\begin{array}{c}v \\
\mathrm{~km} \mathrm{~s}^{-1}\end{array}$ & $\begin{array}{c}\log \xi \\
\left(\mathrm{erg} \mathrm{s} \mathrm{cm}^{-1}\right)\end{array}$ & $\begin{array}{c}N_{\mathrm{H}} \\
10^{21} \mathrm{~cm}^{-2}\end{array}$ & $\begin{array}{c}v \\
\mathrm{~km} \mathrm{~s}^{-1}\end{array}$ & $\begin{array}{c}\text { Cstat/d.o.f. } \\
-\end{array}$ \\
\hline Jan. 01 & $2.60 \pm 0.07$ & $2.2 \pm 0.2$ & $1.2 \pm 0.5$ & $<1600$ & $3.3_{-0.4}^{+0.8}$ & $<17.0$ & $1000 \pm 460$ & $3082 / 2725$ \\
Jan. 05 & $2.41 \pm 0.07$ & $1.5 \pm 0.1$ & $2.3 \pm 0.5$ & $<100$ & $3.2 \pm 0.1$ & $30.0_{-200}^{+110}$ & $<100$ & $1958 / 1435$ \\
July 05 & $2.07 \pm 0.07$ & $1.7_{-0.2}^{+0.3}$ & $3.9_{-1.6}^{+2.6}$ & $<1000$ & $2.8 \pm 0.3$ & $7.6_{-5.5}^{+18}$ & $<1000$ & $1855 / 1437$ \\
\hline
\end{tabular}

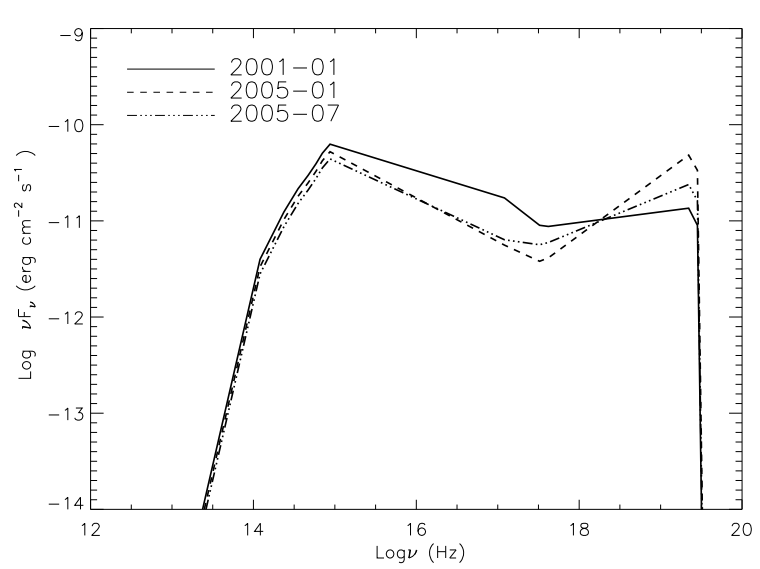

Fig. 1. Spectral energy distribution of Mrk 841 constructed from the $X M M$-Newton data in the optical-X-ray band and from the standard AGN radio-IR continuum included in CLOUDY (see text for more details).

band spectrum. The flux was extracted at $344 \mathrm{~nm}$ for those data sets. At first approximation the complex X-ray unabsorbed broad band continuum can be modelled as a broken power law with an energy break at about $1.5 \mathrm{keV}$ (as derived by a phenomenological fit to the EPIC data, see also Fig. 5 of Petrucci et al. 2007). For each of the three data sets, we took the soft power-law index from the RGS and the harder energy power law index from Petrucci et al. (2007). The very-low energy portion of the SED (radio to IR) is the same as the standard AGN continuum used in CLOUDY (Ferland et al. 1998). The three SEDs are plotted in Fig. 1. Then, we constructed a model for the continuum consisting of an underlying single power law absorbed by warm ionised gas and applied it to the $0.35-1.77 \mathrm{keV}$ data (i.e. the RGS band).

The column density $N_{\mathrm{H}}$ and the ionisation parameter $\xi$ of the gas are free parameters. The ionisation parameter is defined as $\xi=\frac{L}{n_{\mathrm{e}} R^{2}}$, where $L$ is the ionising luminosity in 1-1000 Rydberg, $n_{\mathrm{e}}$ the electron density of the gas, and $R$ the distance of the gas itself from the central source of radiation. The XABS model accounts for the width of the absorption lines through the velocity dispersion parameter. It has been shown in several Seyfert galaxies that UV and X-ray absorbers originate in the same outflowing gas, thus UV observations can in general provide a reliable limit on the X-ray line broadening (Arav et al. 2007; Costantini et al. 2007). Since no constraint on the velocity dispersion of the UV lines is readily available in the literature on Mrk 841 , this parameter was initially fixed to an average value of $20 \mathrm{~km} \mathrm{~s}^{-1}$. When it was left free in the fitting process, it tended to decrease to zero in all the spectra, thus it was eventually kept fixed to $20 \mathrm{~km} \mathrm{~s}^{-1}$. Other free parameters in the model are the photon index of the power law in the RGS band and the outflowing velocity of the warm absorber. The fit statistic improves significantly with the addition of the XABS warm absorber model with respect to the power-law model, but the fit is still not completely satisfactory for all the three epochs.



Fig. 2. Jan. 2005 RGS 2 data modelled with a single component (dashed red line) and a two-component warm absorber (solid blue line). The forbidden emission line of helium-like neon and the RRC of OVII, which are included in both models (see Sect. 3.2), are labelled for clarity.

A close inspection of the spectra shows that absorption features are visible as negative residuals in the neon band (12-14 ̊). The single warm absorber component is not sufficiently ionised to account for absorption in this band. Therefore, a second warm absorber with a higher ionisation level was tested by including in the model a second XABS component. The addition of the second warm absorber leads to a fit statistic improvement by $\Delta C=75$ and $\Delta C=25$ for 3 d.o.f. respectively measured in Jan. and July 2005 spectra. The resulting best-fitting parameters are reported in Table 2. The soft photon index refers to the power law fitted in the RGS band, i.e. 7-35 $\AA$. The quoted parameters and C-statistic refer to the model including the emission features in Table 3. The improvement is less significant in Jan. 2001, but this is not surprising since the column densities in Table 2 indicate that the absorber may be shallower at this epoch. The effect of the second warm absorber is shown in Fig. 2 for Jan. 2005: the Fe UTA is clearly well fitted by the medium ionisation phase, whereas the absorption features of NeIX $1 \mathrm{~s}-3 \mathrm{p}$ at $11.556 \AA$, and $\operatorname{NeX} \operatorname{Ly} \alpha$ at $12.132 \AA$ require a more highly ionised plasma to be accounted for.

The two-phase warm absorber provides a good fit to the data as can be seen in Fig. 3. The main absorption features are identified with transitions of OVI, OVII, OVIII in the 18-23 $\AA$ band, Fe UTA at $15-16 \AA$ and Neon IX-X in the $12-14 \AA$. No significant velocity shift is detected in any of the two warm absorbers (see Table 2), except for the high ionization phase in Jan. 2001. The upper limits are consistent with outflowing absorbers blueshifted by several $\times 10^{2} \mathrm{~km} \mathrm{~s}^{-1}$, as commonly observed in Seyfert 1 Galaxies (e.g. McKernan et al. 2007).

Even with the addition of the second warm absorber, the fit of the Jan. 2005 spectrum is not completely satisfactory, since positive residuals are still present in the OVII band (21-22 $\AA$, see top panel of Fig. 4). The analysis of these residuals in emission is reported in the following section. 


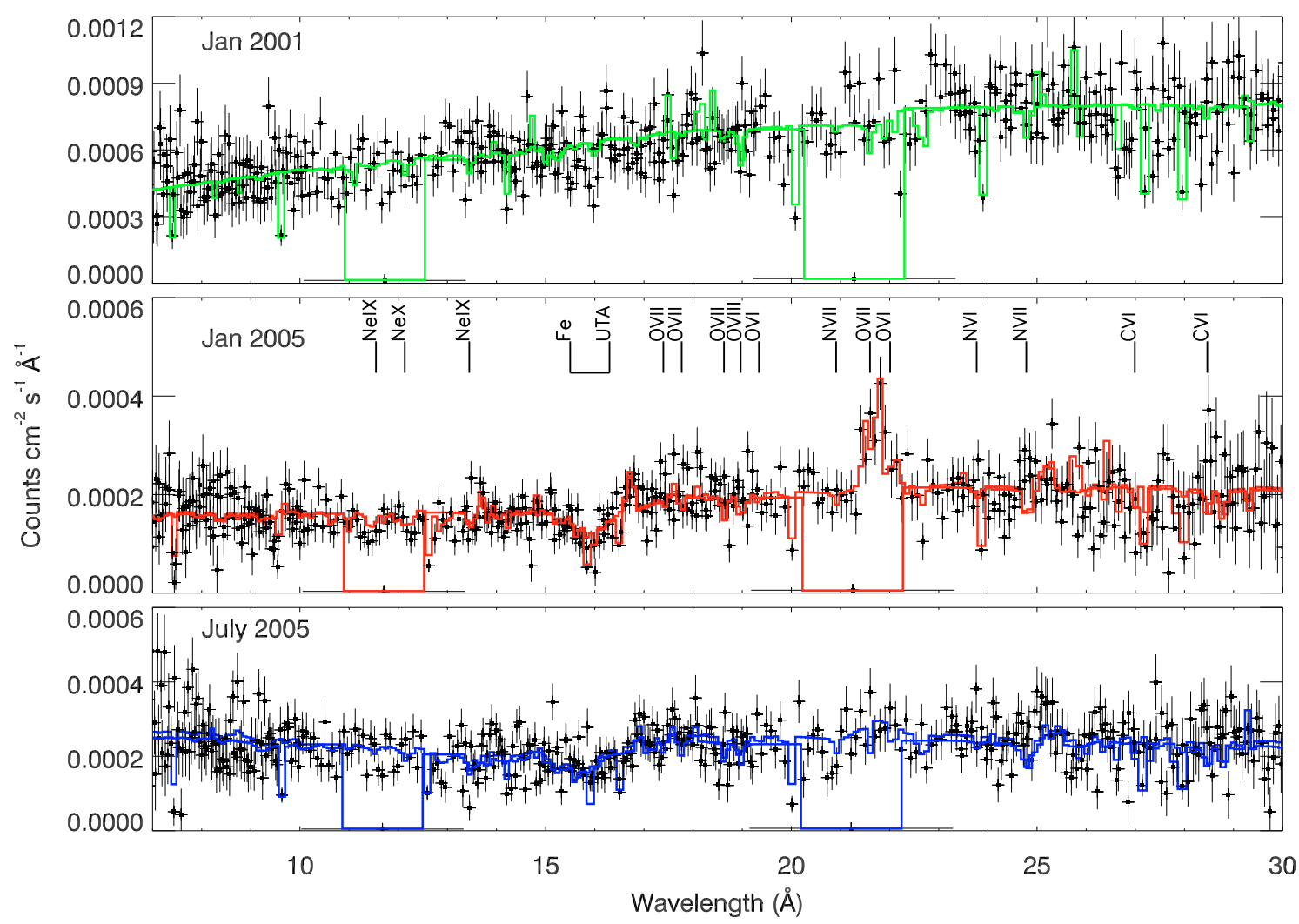

Fig. 3. From top to bottom: RGS rest-frame spectra of Jan. 2001 (for plotting purpose only obsid 0070740101 is shown), Jan. 2005 and July 2005 fitted with the best-fitting model including the components in Tables 2 and 3. For plotting purposes, the spectra are re-binned by a factor of 9 and plotted in the 7-30 ̊ band, where most of the features of interest fall. The labels mark the ionic species with the highest contribution in our warm absorber model.

Table 3. Emission lines parameters and emission measure for RRC in the three epochs.

\begin{tabular}{|c|c|c|c|c|c|c|}
\hline $\begin{array}{c}\text { Obs } \\
-\end{array}$ & $\begin{array}{c}\text { Line } \\
-\end{array}$ & $\begin{array}{c}\lambda \\
(\AA)\end{array}$ & $\begin{array}{c}\text { Flux } \\
\left(10^{-4} \mathrm{ph} \mathrm{cm}^{-2} \mathrm{~s}^{-1}\right)\end{array}$ & $\begin{array}{c}\Delta C^{1} \\
-\end{array}$ & $\begin{array}{c}F W H M \\
(\AA)\end{array}$ & $\begin{array}{l}\text { RRC EM } \\
\left(10^{58} \mathrm{~cm}^{-3}\right)\end{array}$ \\
\hline \multirow{5}{*}{ Jan. 01} & OVII (r) & 21.600 & $<0.42$ & - & 0-width & \multirow{5}{*}{$\begin{array}{l}\text { OVII }<450 \\
\text { CVI }<4300\end{array}$} \\
\hline & OVII (i) & 21.790 & $<0.15$ & - & 0-width & \\
\hline & OVII (f) & 22.101 & $<0.43$ & - & 0-width & \\
\hline & NeIX (f) & 13.699 & $<0.12$ & - & 0-width & \\
\hline & OVII (gau) & 21.7 & $<0.85$ & - & 0.55 & \\
\hline \multirow{5}{*}{ Jan. 05} & OVII (r) & 21.600 & $0.24 \pm 0.16$ & 17 & - & \multirow{5}{*}{$\begin{array}{c}\mathrm{OVII}=1500 \pm 800 \\
\mathrm{CVI}=2500 \pm 1800 \\
\Delta C \text { stat }=23\end{array}$} \\
\hline & OVII (i) & 21.790 & $0.26_{-0.16}^{+0.26}$ & 16 & - & \\
\hline & OVII (f) & 22.101 & $<0.20$ & - & - & \\
\hline & NeIX (f) & 13.699 & $0.10 \pm 0.05$ & 10 & - & \\
\hline & OVII (gau) & $21.75_{-0.36}^{+0.23}$ & $0.64 \pm 0.53$ & 18 & $0.60_{-0.35}^{+0.52}$ & \\
\hline \multirow{5}{*}{ July 05} & OVII (r) & 21.600 & $<0.04$ & - & - & \multirow{5}{*}{$\begin{array}{c}\mathrm{OVII}=1860 \pm 1000 \\
\mathrm{CVI}=2500 \pm 2300 \\
\Delta C \text { stat }=15\end{array}$} \\
\hline & OVII (i) & 21.790 & $<0.25$ & - & - & \\
\hline & OVII (f) & 22.101 & $<0.23$ & - & - & \\
\hline & NeIX (f) & 13.699 & $<0.15$ & - & - & \\
\hline & OVII (gau) & $21.71 \pm 0.23$ & $0.51 \pm 0.38$ & 6 & $0.54_{-0.44}^{+0.57}$ & \\
\hline
\end{tabular}

Notes. ${ }^{(1)}$ When $\Delta C$ stat $\leq 3$, the upper limits on the line flux are reported.

\subsection{Emission features in the RGS spectra}

\subsubsection{Narrow emission lines}

We start by fitting Jan. 2005 with three narrow-line components at the wavelengths corresponding to the rest frame positions of the resonance, intercombination, and forbidden lines $(r$, $i, f)$ in the OVII line triplet at $21.600,21.790$, and $22.101 \AA$, respectively. The analysis of emission lines in this portion of the spectrum is complex because of the superposition of the absorption lines imprinted by the warm absorber. The peak wavelengths of the three lines are kept frozen to the laboratory values. Since the $r$ transition has a large oscillator strength in the absorption component, most of the emission line at this wavelength will be re-absorbed, making a precise measure of its flux somewhat difficult. The $i$ and $r$ components are detected, while only an upper limit is found for the $f$ line. Less prominent positive residuals are present in the NeIX band, hence we searched for emission 

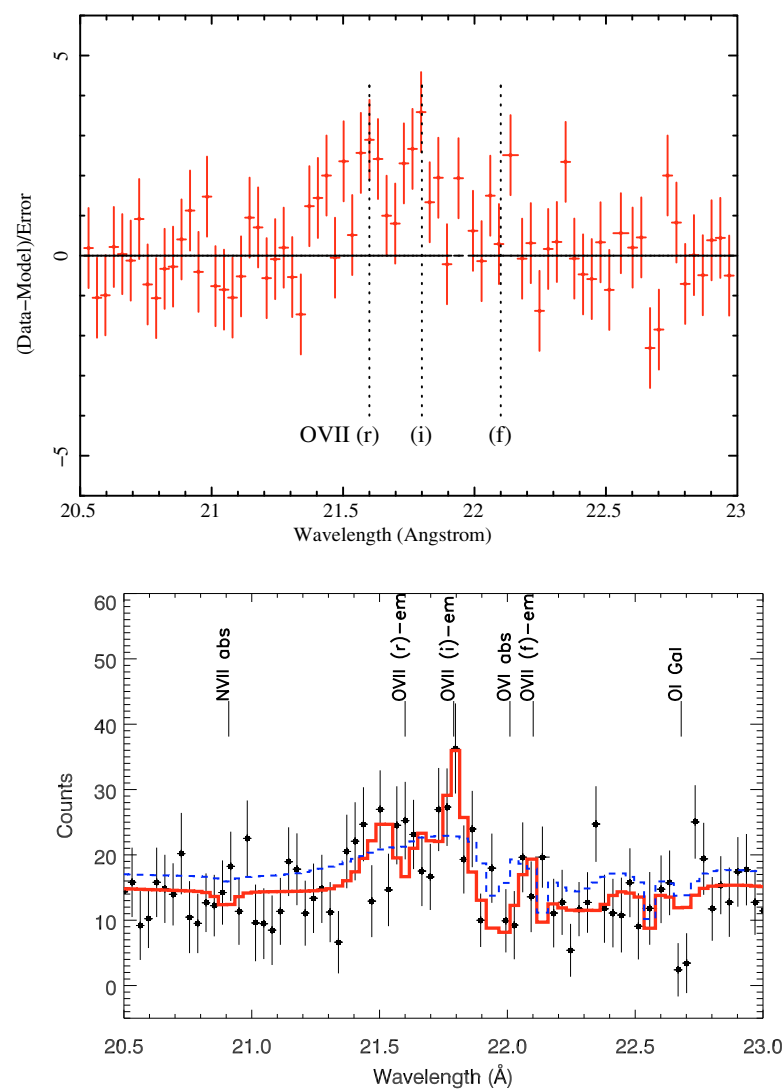

Fig. 4. Top: residuals of the continuum model in the OVII band. Bottom: zoom on the OVII triplet in Jan. 2005. Line parameters are reported in Table 3. The solid red line corresponds to the best-fitting model (including the emission lines in Table 3 and the underlying warm absorber in Table 2). The dashed blue line shows the underlying OVII broad Gaussian line after removing the contribution of the narrow lines triplet and the warm absorber.

lines also in this portion of the spectrum. Only the NeIX forbidden component at $13.699 \AA$ is detected (the neon band is shown in Fig. 2). The emission lines fluxes and significances in terms of $\mathrm{C}$ statistic improvement for one free parameter, are reported in Table 3. The structure of the line triplet fitted in Jan. 2005 data is plotted in Fig. 4 (thick red line).

We model the spectra of Jan. 2001 and July 2005 with the same emission line components included for Jan. 2005. Not surprisingly, given that the continuum flux is higher in these two data sets (see Table 1), we could measure only the upper limits on the line fluxes, which are generally consistent with Jan. 2005 spectra, except for the OVII resonance line in July 2005 that may be severely underestimated.

\subsubsection{Radiative recombination continua}

The spectrum of Jan. 2005 displays an additional emission feature around $17 \AA$, consistent with the position of the radiative recombination continuum (RRC) of OVII (see Fig. 2 for a closeup of this feature). A closer look at longer wavelengths suggests that other RRC components from Carbon may give significant contribution in the positive residuals of the data. In our analysis, these features are fitted with the RRC model in SPEX. The width of the RRC profiles is a very sensitive indicator of the electron temperature in the recombining plasma (Liedahl \& Paerels 1996; Liedahl 1999). Our data suggest the presence of a narrow RRC at the OVII wavelength (see Fig. 2), indicative of temperature of few $\times 10^{4} \mathrm{~K}$. Therefore, we fit the RRC emission measure ${ }^{2}$ of OVII and CVI as free parameters assuming an electron temperature corresponding to $k T=3 \mathrm{eV}$, i.e. $T=3.5 \times 10^{4} \mathrm{~K}$. If the temperature is left free to vary in the fit, we find $k T=2.8_{-1.4}^{+2.9} \mathrm{eV}$.

The improvement in the fit statistic for adding the RRC component in Jan. 2005 is $\Delta C=23$ for 2 degrees of freedom. The OVII RRC seems to be slightly redshifted from its laboratory position; we applied a small redshift of $z=0.001$ to match the observed excess in the data. The same was done for the C VI RRC around $25 \AA$, where the shift is less evident due to the lower signal-to-noise of our spectra in this band. As described in the previous section for the narrow emission lines, we included the RRC in the model for all the three spectra and reported the bestfitting parameters in Table 3.

\subsubsection{Broad oxygen line}

Once the narrow lines are included in the fit, the residuals around the OVII triplet in Jan. 2005 still show some structure in excess, particularly on the left side of the resonant line. To account for these residuals, a broad Gaussian line was added in the model with width and wavelength free to vary. The resulting best-fitting parameters for this component are $\lambda=21.75_{-0.36}^{+0.23} \AA$, $F W H M=0.60_{-0.35}^{+0.52} \AA$, and flux $=0.64 \pm 0.53 \times 10^{-4}$ photons $\mathrm{cm}^{-2} \mathrm{~s}^{-1}$. The addition of the broad line component leads to an improvement of $\Delta C$ stat $=18$ for 3 degrees of freedom, corresponding to a significance level higher than $99 \%$. Figure 4 shows the separate contribution of the Gaussian profile to the best fit model for the OVII line triplet. For simplicity, the broad line at $21.7 \AA$ will only be referred to as "OVII line", without identifying a specific transition. The same line component was included in July 2005 yielding the following best-fitting parameters: $\lambda=21.71 \pm 0.23 \AA, F W H M=0.54_{-0.44}^{+0.57} \AA$ and flux $=0.51 \pm 0.38 \times 10^{-4}$ photons $\mathrm{cm}^{-2} \mathrm{~s}^{-1}$. Although the significance of this component in July 2005 (around 90\%) is lower than in Jan. 2005, the generally good agreement in the bestfitting parameters for the two spectra seem to support the presence of the same line in July 2005. In Jan. 2001 data, we added the broad component as we did for the narrow lines. Since the higher continuum flux prevents any determination of the line properties, to measure the line flux, the position and width of the Gaussian component have been fixed to the average value found for the 2005 epochs (see Table 3 ). An upper limit of $<0.85$ photons $\mathrm{m}^{-2} \mathrm{~s}^{-1}$ (consistent with the 2005 measurements) was found for the broad component in 2001.

\section{Discussion}

The analysis of the RGS data of Mrk 841 presented herein provides a considerable amount of information. A large contribution from a warm absorber consisting of two ionisation components has been revealed and most interestingly, it proves to be relatively stable on a time scale of 4 years. In addition, emission features from highly ionised species of neon, oxygen, and carbon have been ascertained. These topics will be addressed separately in the following sections. The discussion is based on the results from the global best fit (i.e. absorption+emission), which is reported in Tables 2 and 3 .

\footnotetext{
2 The emission measure corresponds to $n_{\mathrm{e}} n_{\text {ion }} V$, where $n_{\mathrm{e}}$ is the gas electron density, $n_{\text {ion }}$ the density of the parent ion that recombines to the ground state, and $V$ the emitting volume.
} 


\subsection{A two-phase warm absorber in Mrk 841}

The properties of the ionised absorber emerging from the spectral fits described in Sect. 3.1, indicate that there are two distinct levels of ionisation in the absorbing gas. A medium-phase warm absorber characterised by $\log \xi$ in the range $1.5-2.2 \mathrm{erg} \mathrm{s} \mathrm{cm}^{-1}$ and column density of the order of $10^{21} \mathrm{~cm}^{-2}$ is responsible for transition in the Fe UTA and in the oxygen band.

The availability of spectra from different epochs may allow a variability study of the absorber physical conditions, particularly considering that because the Fe UTA is the result of a blend of absorption lines arising from several iron ions, its shape and position are very sensitive to changes in the ionisation balance of the gas and, ultimately, to luminosity variations of the source (Behar et al. 2001). The errors in Table 2 show that $\xi$ and $N_{\mathrm{H}}$ are generally consistent through the three data sets, although there seems to be a decrease in ionisation accompanied by a moderate increase of the column density going from 2001 to Jan. 2005. Indeed, the Fe UTA is much more evident in both 2005 spectra because the lower ionisation state observed at this epoch is able to produce a high number of transitions in Fe XV-XVII in the 15-16 ̊ region. According to our model, absorption from OVI and OVII is also strong in this source. These absorption lines are not easily recognisable, especially in Jan. 2005 spectrum, due to the presence of the OVII emission line triplet. The OVI-OVII absorption is likely associated to the gas component in a medium ionisation state.

The higher ionisation phase $\left(\log \xi \sim 3 \mathrm{erg} \mathrm{s} \mathrm{cm}^{-1}\right)$ is instead responsible for imprinting absorption at shorter wavelengths mainly in the neon band around 12-14 $\AA$. This component is characterised by a column density of the order of $10^{22} \mathrm{~cm}^{-2}$, much higher than the one measured in the medium phase. In principle, warm gas at this ionisation level can produce absorption lines in the Fe $K$ band around 6-7 keV, which may play a key role in shaping the Fe $\mathrm{K}$ alpha line profile (e.g. Revees et al. 2004; Turner \& Miller 2009). Indeed, Longinotti et al. (2004) and Petrucci et al. (2007) reported peculiar behaviour in the Fe $\mathrm{K} \alpha$ line in their previous analysis of Mrk 841, but none of these works included detailed modelling of the warm absorber. The detection of a high ionisation warm absorber in the RGS data prompted us to test for absorption effects on the Fe K line. This check was performed by simply applying our best-fitting model to the EPIC-pn data of Jan. 2005, i.e., the spectrum with the highest column density in the high ionisation phase (Table 2). With the parameters frozen to the values of Table 2, we found that this warm absorber component is not deep enough for introducing any additional curvature around the Fe line, or for modifying the line profile itself.

\subsection{Density of the warm absorber}

In about four-years time, the low-energy flux of Mrk 841 decreased by a factor of 3.5 (Table 1). In Jan. 2005, when the source was fainter, we measured also a lower ionisation parameter for the cold phase, while the column density stayed constant within the errors (Table 2). Given the uncertainty on the outflow velocity, we assume here that we detected the same gas material in both 2001 and 2005, albeit with different ionisations.

We estimated a lower limit for the density of such gas, assuming a recombination time of 1422 days (4 years time between Jan. 2001 and Jan. 2005), i.e. $t_{\mathrm{s}}=\frac{t_{\mathrm{o}}}{1+z}$ in the source rest frame. We considered all the Fe ions detected in the spectrum. Each ion has its own recombination time, which is inversely related to the

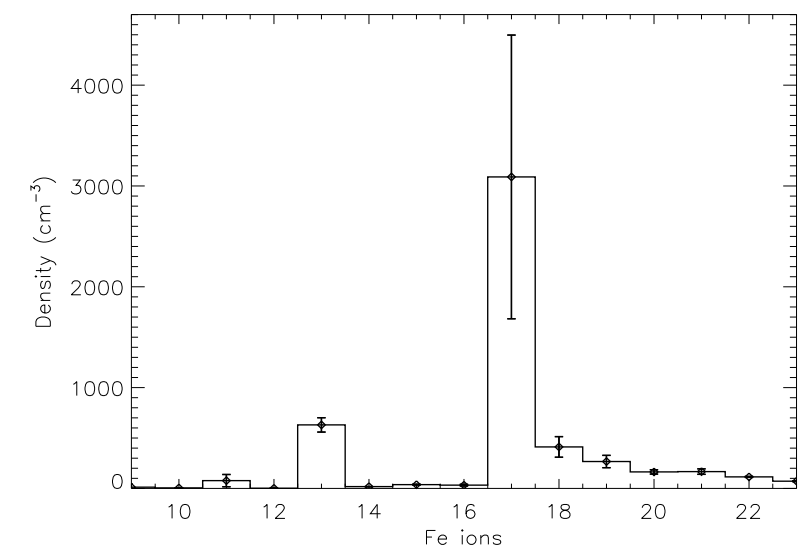

Fig. 5. Electron density in the warm absorber estimated using the recombination of the iron ions produced by the gas with $\log \xi=2.2 \pm 0.23$ and $N_{\mathrm{H}} \sim 1.2 \pm 0.5 \times 10^{21} \mathrm{~cm}^{-2}$.

gas density $n_{\mathrm{e}}$, as reported in Bottorff et al. (2000) and Detmers et al. (2008):

$t\left(X_{i}\right)=\left(\alpha_{r}\left(X_{i}\right) n_{\mathrm{e}}\left[\frac{f\left(X_{i+1}\right)}{f\left(X_{i}\right)}-\frac{\alpha_{r}\left(X_{i-1}\right)}{\alpha_{r}\left(X_{i}\right)}\right]\right)^{-1}$

where $t\left(X_{i}\right)$ is the recombination timescale of the ion $X_{i}, \alpha\left(X_{i}\right)$ is the recombination rate from ion $X_{i=1}$ to ion $X_{i}$, and $f\left(X_{i}\right)$ represents the fraction of the element $X$ at the ionisation state $i$.

This relation allows us to put further constraints on the lower limit of the density. We used the values predicted by the best-fit global modelling of the absorber, as the quality of the data does not allow a line-by-line fitting on the Fe ions to be performed, especially in the crowded region of the iron UTA. The associated error on the ionic column densities is therefore the scaled value of the error on the total $N_{\mathrm{H}}$.

The gas density is a function of the recombination rates, which are in turn a function of the gas temperature $T_{\mathrm{e}}$, before recombination (Bottorff et al. 2000; Detmers et al. 2008). The value of $T_{\mathrm{e}}$ is linked to the ionisation parameter $\xi$ via the ionisation balance. The error on $\xi$ (Table 2), rather than the error on individual ionic column densities, has the major weight in the density determination. The radiative recombination rates for iron were analytically determined using the expression and recombination coefficient provided in Woods et al. (1981).

In Fig. 5 we show the density lower limits for the Fe ions. The ion that provides the higher value for $n=3.1 \pm 1.4 \times 10^{3} \mathrm{~cm}^{-3}$ is Fe XVII.

\subsection{On the origin of the narrow emission lines and radiative recombination continua}

The following discussion is based chiefly on the findings for Jan. 2005 spectrum, where the emission line components are detected with highest significance. The narrowness of the RRC profiles is consistent with an electron temperature of a few $\times 10^{4} \mathrm{~K}$, and it seems to indicate that photoionisation from the central source is the primary mechanism acting on the surrounding gas (e.g. Kinkhabwala et al. 2002; Armentrout et al. 2007).

The relative intensity of line triplets in He-like ions provides an excellent diagnostic of the physical properties of the emitting plasma (Porquet \& Dubau 2000, and reference therein). In general, it has been observed that photoionisation processes in Seyfert Galaxies give rise to line triplets dominated by the forbidden transition (e.g. NGC 1068, Kinkhabwala et al. 2002). If 
we consider the line fluxes of the OVII triplet in Mrk 841, we note that their ratio is not typical of photoionisation, the less prominent line being the forbidden transition, as is also evident from Fig. 4. The intercombination component at $21.790 \AA$ is as strong as the resonance one, if not higher: the presence of a bad pixel between the $r$ and $i$ lines does not allow precise reconstruction of the $r$ emission line. On the contrary, we measure an upper limit for the forbidden component. Since this line is not affected by instrumental effects and is generally expected to be dominant in AGN spectra, this measurement provides a stringent limit to be considered in the discussion of the line ratios.

The $R$ ratio $\frac{f}{i}$ provides a compelling probe of the gas density in photoionised plasma (see Porquet \& Dubau 2000). The low value of this ratio $(R<0.76)$ in Mrk 841 points to emission from a gas at very high density, i.e. $n_{\mathrm{e}} \geq 10^{11} \mathrm{~cm}^{-3}$. This result partly recalls the case of the Seyfert 1 Galaxy Mrk 335 that displayed a photoionised OVII triplet with a dominant $i$ line during a particularly low flux state (Longinotti et al. 2008).

The $G$ ratio $\frac{f+i}{r}$ instead is often used as an estimator of the electron temperature in the gas. We can estimate an upper limit on the $G$ ratio by taking the upper limits on the three components of the triplet into account (i.e., the upper error bars for the two lines for which they are available and the upper limit on the $f$ line). In this way, the $G$ ratio is estimated to be less than 1.8. This limit is somewhat intermediate between a pure collisional and a pure photoionisation-dominated plasma (see Fig. 7 in Porquet \& Dubau 2000), possibly indicating that mixed processes are at work. We note some discrepancy between the temperature of the recombining plasma inferred in this work by the RRC width $\left(\sim 10^{4} \mathrm{~K}\right)$ and the temperature prescribed for the hybrid plasma by the calculations reported in Porquet \& Dubau (2000). However, as highlighted for Mrk 3 (Sako et al. 2000) and NGC 4151 (Armentrout et al. 2007), the intensity of resonance lines in He-like triplets could be enhanced by photo-excitation mechanisms induced by the AGN, which leads to a decrease in the observed $G$ ratio (Godet et al. 2004). In principle, this effect could be tested in our data by accounting for microturbulence in the resonance line (see Armentrout et al. 2007), but we recall that the velocity dispersion was undetermined even for the warm absorber lines (less than $20 \mathrm{~km} \mathrm{~s}^{-1}$, see Sect. 3.1), thus not easily determined with the present instrumental resolution.

\subsection{One photoionisation region for the warm absorber and the warm mirror?}

While certainly very interesting, the soft X-ray properties in Mrk 841 are not unique because several AGNs show features from ionised plasma both in absorption and emission e.g. NGC 4151, (Armentrout et al. 2007; Kraemer et al. 2005) and NGC 3783 (Behar et al. 2003). For some cases, attempts are being made to ascribe the two processes to the same photoionised plasma (e.g. NGC 4051, Nucita et al. submitted). The main unknown is the geometry of the circumnuclear gas, which should act as a "warm mirror" for scattering the emission lines and, at the same time, it should intercept the observer's line of sight for producing warm absorber features.

For a large number of X-ray obscured Seyfert galaxies, there are several indications that the $\mathrm{X}$-ray photoionisation region where the bulk of soft X-ray line emission takes place, is spatially coincident with the optical narrow line region (NLR) (see Bianchi et al. 2006; and Guainazzi \& Bianchi 2007). Seyfert 1 objects, with unobscured X-ray spectra, offer instead a direct view of the continuum source and the surrounding high density gas giving rise to optical broad lines (i.e. the broad line region (BLR)), therefore introducing more diversity in determining the distribution of the hot plasma. As an example, in the Seyfert 1 NGC 4051 (Pounds et al. 2004) and NGC 5548 (Kaastra et al. 2002; Detmers et al. 2008), the gas density inferred from the observed emission lines indicates an origin in the NLR, whereas in Mrk 335, the high density line-emitting gas $\left(n_{\mathrm{e}}=10^{9-11} \mathrm{~cm}^{-3}\right)$ was located within the source BLR (Longinotti et al. 2008).

The present study of Mrk 841 with the detection of an important warm absorber component and photoionised emission lines, provides the opportunity to compare and, possibly, link their properties. The ionisation parameter of the photoionised gas $\xi=\frac{L}{n_{\mathrm{e}} R^{2}}$ depends on the plasma electron density, its radial distance from the source of radiation, and the ionising luminosity. An average luminosity of $10^{45} \mathrm{erg} \mathrm{s}^{-1}$ was estimated from the SED in Fig. 1 by integrating the data of Jan. 2001 and Jan. 2005 over 1-1000 Rydberg.

In Sect. 4.2, we derived a lower limit for the density of the warm absorber $\left(>10^{3} \mathrm{~cm}^{-3}\right)$, which can loosely constrain the distance to $<$ few tens of pc. Nevertheless, this constraint can be refined by using the limit on the density inferred by the OVII triplet $\left(n_{\mathrm{e}} \geq 10^{11} \mathrm{~cm}^{-3}\right)$, under the assumption that the emission lines originate within a gas at the same ionisation state of the warm absorber gas. Our two-phase model for Jan. 2005 yields $\log \xi \sim 1.5$ and $3.2 \mathrm{erg} \mathrm{s} \mathrm{cm}^{-1}$ (see Table 2). However, in the model of the gas with $\log \xi \sim 3.2 \mathrm{erg} \mathrm{s} \mathrm{cm}^{-1}$ the column density of OIX is much higher than the one of OVII and OVIII, therefore such a highly ionised gas is unlikely to produce OVII emission. Assuming the ionisation parameter of the low phase, the radial distance $R$ of the "warm emitter gas" is then constrained to less than $1.6 \times 10^{16} \mathrm{~cm}$.

The person who wishes to put this result in the context of the source may consider that the optical BLR lies at a radius of about $4.4 \times 10^{16} \mathrm{~cm}$, as roughly estimated from the $F W H M$ of $5500 \mathrm{~km} \mathrm{~s}^{-1}$ in the optical lines (Boroson \& Green 1992). Thus, the ionised gas seems to encompass the BLR, analogously to Mrk 335 (Longinotti et al. 2008). We checked the agreement of the optical FWHM and the width of the OVII line triplet by fitting the three components with a Gaussian profile and freezing the rest of the model. We found the following $F W H M$ for $r, i, f$, respectively: $400 \pm 60,<1400$ and $<5300 \mathrm{~km} \mathrm{~s}^{-1}$. While the velocities in the $i$ and $f$ components can still be reconciled with BLR, it is hard to explain the apparent inconsistency of the resonant line with the rest of the triplet. We note, however, that the reconstruction of the $r$ line is rather complex, as highlighted in Sect. 3.2.1. A more sophisticated modelling of the emission lines in high signal-to-noise data would be needed to supplement our hypothesis.

A few words must be said on the line fluxes in the other two data sets for which only upper limits are available. The apparent lack of strong emission lines in Jan. 2001 and Jan. 2005 can be phenomenologically explained as an effect of the increased continuum flux. As a consequence, the emission component is swamped and covered in the observed spectra. While it is hard to exclude intrinsic variability in the emission line fluxes, especially since the continuum flux varies, there is no compelling evidence for variation in the present data.

\subsection{The broad OVII line in the RGS data}

The broad emission line around $\sim 21.7 \AA$ is detected with high significance in both 2005 observations. If fitted with a Gaussian profile, the $F W H M$ corresponds to $\sim 8200_{-4700}^{+7200} \mathrm{~km} \mathrm{~s}^{-1}$, 
for Jan. 2005. Considering the large error bars, this velocity is consistent with the optical $\mathrm{H} \beta F W H M$ of $5500 \mathrm{~km} \mathrm{~s}^{-1}$ reported by Boroson \& Green (1992). Broad emission features in the soft X-ray spectra of Seyfert 1 Galaxies has been reported in the Chandra-LETG of Mrk 279 (Costantini et al. 2007), NGC 5548 (Steenbrugge et al. 2005), NGC 4051 (Steenbrugge et al. 2009), and in the XMM-Newton-RGS spectra of NGC 4051 (Ogle et al. 2004; Ponti et al. 2006). In most of these cases, the line profiles were consistent with Gaussians, and in the first two sources, the velocity width of the emission lines was in agreement with measurements of UV lines in the BLR.

In the past, it was proposed to model RGS spectral lines from H-like ions in the Seyfert 1 Galaxies of MCG-6-30-15 and Mrk 766 with a relativistic profile (Branduardi-Raymont et al. 2001). We tried to convolve the OVII Gaussian emission line in Mrk 841 with a relativistic profile using the LAOR kernel (Laor 1991) in Jan. 2005 spectrum. No good fit was found, perhaps not surprisingly since the data quality of Mrk 841 is not comparable to the long-integration RGS spectra of the two bright sources mentioned above, keeping the test for relativistic broadening from being viable in this source.

Considering the agreement of the OVII and $\mathrm{H} \beta$ lines $F W H M$ in Mrk 841, a BLR origin for the OVII broad line is favoured. Unfortunately, the lack of simultaneous UV spectral data for this source prevents us from performing a more detailed analysis of this feature and its relation to the multi-wavelength properties.

\section{Summary of conclusions}

In the following we summarise the main results of the first analysis of Mrk 841 high-resolution X-ray spectra:

- Warm absorber: a two-phase warm absorber with $\log \xi \sim$ 1.5-2.2 $\mathrm{erg} \mathrm{s} \mathrm{cm}^{-1}$ and $\log \xi \sim 3 \mathrm{erg} \mathrm{s} \mathrm{cm}^{-1}$ was observed in the three spectra from 2001 through 2005 , a moderate decrease in the ionisation state was observed going from the brightest flux state (2001) to the dimmer state (2005), no outflow was detected in the data and the high ionisation component has no effect on the Fe $K$ band.

- Emission features: narrow OVII and NeIX emission lines were detected in the Jan. 2005 spectrum, the line ratio in the OVII triplet points to an origin in a gas at high electron density $\left(n_{\mathrm{e}} \geq 10^{11} \mathrm{~cm}^{-3}\right)$, the presence of narrow RRC from OVII and CVI is consistent with photoionisation processes, if it is assumed that the emission component and the warm absorber originate within a gas at the same ionisation state, the inferred distance for the emission lines is constrained within $10^{16} \mathrm{~cm}$ from the central source.

- Broad Gaussian: a broad Gaussian line centred at $21.7 \AA$ was interpreted as emission from OVII, the FWHM is fully compatible with that of the optical broad emission lines in Mrk 841.

Acknowledgements. This paper is based on observations obtained with the $X M M-N e w t o n$ satellite, an ESA science mission with instruments and contributions directly funded by ESA Member States and NASA. We acknowledge support from the Faculty of the European Space Astronomy Centre (ESAC) and from the French GDR PCHE for financially supporting the collaboration meetings needed to finalise this work. G.P. thanks ANR for support (ANR-06-JCJC-0047).

\section{References}

Anders, E., \& Grevesse, N. 1989, Geochim. Cosmochim. Acta, 53, 197 Arav, N., et al. 2007, ApJ, 658, 829

Armentrout, B. K., Kraemer, S. B., \& Turner, T. J. 2007, ApJ, 665, 237

Behar, E., Sako, M., \& Kahn, S. M. 2001, ApJ, 563, 497

Behar, E., Rasmussen, A. P., Blustin, A. J., et al. 2003, ApJ, 598, 232

Bianchi, S., Matt, G., Haardt, F., et al. 2001, A\&A, 376, 77

Bianchi, S., Matt, G., Balestra, I., Guainazzi, M., \& Perola, G. C. 2004, A\&A, 422,65

Bianchi, S., Guainazzi, M., \& Chiaberge, M. 2006, A\&A, 448, 499

Blustin, A. J., Page, M. J., Fuerst, S. V., Branduardi-Raymont, G., \& Ashton, C. E. 2005, A\&A, 431, 111

Boroson, T. A., \& Green, R. F. 1992, ApJS, 80, 109

Bottorff, M. C., Korista, K. T., \& Shlosman, I. 2000, ApJ, 537, 134

Branduardi-Raymont, G., Sako, M., Kahn, S. M., et al. A\&A, 365, L140

Cash, W. 1979, ApJ, 228, 939

Costantini, E., et al. 2007, A\&A, 461, 121

Crenshaw, D. M., Kraemer, S. B., \& George, I. M. 2003, ARA\&A, 41, 117

Crummy, J., Fabian, A. C., Gallo, L., \& Ross, R. R. 2006, MNRAS, 365, 1067 den Herder, J. W., et al. 2001, A\&A, 365, L7

Detmers, R. G., Kaastra, J. S., Costantini, E., McHardy, I. M., \& Verbunt, F. 2008, A\&A, 488, 67

Dickey, J. M., \& Lockman, F. J. 1990, ARA\&A, 28, 215

Ferland, G. J., Korista, K. T., Verner, D. A., et al. 1998, PASP, 110, 761

Gabel, J. R., et al. 2005, ApJ, 631, 741

George, I. M., Nandra, K., Fabian, A. C., et al. 1993, MNRAS, 260, 111

Gierliński, M., \& Done, C. 2004, MNRAS, 349, L7

Godet, O., Collin, S., \& Dumont, A.-M. 2004, A\&A, 426, 767

Guainazzi, M., \& Bianchi, S. 2007, MNRAS, 374, 1290

Kaastra, J. S., Mewe, R., \& Nieuwenhuijzen, H. 1996, UV and X-ray Spectroscopy of Astrophysical and Laboratory Plasmas, 411

Kaastra, J. S., Steenbrugge, K. C., Raassen, A. J. J., et al. 2002, A\&A, 386, 427

Kaspi, S., et al. 2001, ApJ, 554, 216

Kinkhabwala, A., et al. 2002, ApJ, 575, 732

Kraemer, S. B., et al. 2005, ApJ, 633, 693

Krongold, Y., Nicastro, F., Brickhouse, N. S., Elvis, M., \& Mathur, S. 2005, ApJ, 622,842

Krongold, Y., Nicastro, F., Elvis, M., et al. 2007, ApJ, 659, 1022

Laor, A. 1991, ApJ, 376, 90

Liedahl, D. A. 1999, X-Ray Spectroscopy in Astrophysics, Lecture Notes Phys., 520,189

Liedahl, D. A., \& Paerels, F. 1996, ApJ, 468, L33

Longinotti, A. L., Nandra, K., Petrucci, P. O., \& O’Neill, P. M. 2004, MNRAS, 355,929

Longinotti, A. L., Nucita, A., Santos-Lleo, M., \& Guainazzi, M. 2008, A\&A, 484,311

McKernan, B., Yaqoob, T., \& Reynolds, C. S. 2007, MNRAS, 379, 1359

Nandra, K., Turner, T. J., George, I. M., et al. 1995, MNRAS, 273, 85

Nandra, K., George, I. M., Mushotzky, R. F., Turner, T. J., \& Yaqoob, T. 1997, ApJ, 477, 602

Netzer, H., Chelouche, D., George, I. M., et al. 2002, ApJ, 571, 256

Ogle, P. M., Mason, K. O., Page, M. J., et al. 2004, ApJ, 606, 151

Petrucci, P. O., et al. 2002, A\&A, 388, L5

Petrucci, P. O., et al. 2007, A\&A, 470, 889

Ponti, G., Miniutti, G., Cappi, M., et al. 2006, MNRAS, 368, 903

Porquet, D., \& Dubau, J. 2000, A\&AS, 143, 495

Porquet, D., Mewe, R., Dubau, J., Raassen, A. J. J., \& Kaastra, J. S. 2001, A\&A, 376,1113

Pounds, K. A., Reeves, J. N., King, A. R., \& Page, K. L. 2004, MNRAS, 350, 10

Reeves, J. N., Nandra, K., George, I. M., et al. 2004, ApJ, 602, 648

Reynolds, C. S. 1997, MNRAS, 286, 513

Sako, M., Kahn, S. M., Paerels, F., \& Liedahl, D. A. 2000, ApJ, 543, L115

Sako, M., et al. 2001, A\&A, 365, L168

Steenbrugge, K. C., et al. 2005, A\&A, 434, 569

Steenbrugge, K. C., Fenovčík, M., Kaastra, J. S., Costantini, E., \& Verbunt, F. 2009, A\&A, 496, 107

Véron-Cetty, M.-P., \& Véron, P. 2001, A\&A, 374, 92

Turner, T. J., \& Miller, L. 2009, A\&ARv, 17, 47

Weaver, K. A., Gelbord, J., \& Yaqoob, T. 2001, ApJ, 550, 261

Woods, D. T., Shull, J. M., \& Sarazin, C. L. 1981, ApJ, 249, 399 